\title{
1 ECOFEMINISMO Y DERECHOS \\ DE LA NATURALEZA. CRUCES ENTRE LEY, ESTADO Y SENSIBILIDADES
}

ECOFEMINISM AND RIGHTS OF NATURE. INTERSECTIONS BETWEEN LAW, STATE AND SENSIBILITIES

\author{
Pablo Pereira $^{1}$; Laura Borsellino ${ }^{2}$
}

RESUMEN / En este ensayo presentamos una serie de reflexiones centradas en los derechos de la naturaleza (DDNN) y los ecofeminismos, motivados por los signos actuales de violencia contra cuerpos y territorios en América Latina y el Caribe. Sostendremos que si bien abren unas vías de indagación más que interesantes al disputar lo establecido por las leyes e instituciones modernas, las visiones ecocéntricas y biocéntricas existentes, materializadas en los DDNN, se encuentran atravesadas por la desigualdad de género. Así, promovemos una mirada más crítica contemplando que se comienza desde una posición desigual y jerárquica -basada en la construcción social del género-, aún subyacente en la formulación discursiva de las leyes y en la amplitud de reconocimientos que efectúa. Al proponer una sinergia entre Ios DDNN y el ecofeminismo, pretendemos ampliar el discurso legal con una práctica teórica y una sensibilidad que lo trascienda.

PALABRAS CLAVE / Derechos de la Naturaleza, Ecofeminismo, Estado, sensibilidades, ética.
ABSTRACT / In this essay we present a series of reflections focused on the rights of nature (RoN) and ecofeminisms, motivated by the current signs of violence against bodies and territories in Latin America and the Caribbean. We will argue that although they open more than interesting paths of inquiry by disputing what is established by modern laws and institutions, the existing ecocentric and biocentric visions, materialized in the RoN, are traversed by gender inequality. Thus, we promote a more critical view, contemplating that it starts from an unequal and hierarchical position -based on the social construction of gender-, still underlying in the discursive formulation of laws and in the wide range of recognitions it makes. By proposing a synergy between the RoN and ecofeminism, we intend to expand the legal discourse with a theoretical practice and a sensibility that transcends it.

KEY WORDS / Rights of Nature, Ecofeminism, State, sensibilities, ethics.

(1) Universidad Nacional de Buenos Aires, Argentina. ppereira@sociales.uba.ar

(2) Universidad Nacional de Buenos Aires, Argentina. laura.borse@gmail.com 


\section{Introducción}

En este ensayo presentamos una serie de reflexiones centradas en los derechos de la naturaleza (DDNN) y los ecofeminismos, motivados por los signos actuales de violencia contra cuerpos y territorios en América Latina y el Caribe. Es posible evaluar, un poco a la distancia, algunas experiencias de las últimas décadas en países como Ecuador y Bolivia, que incorporaron en sus textos constitucionales el reconocimiento de derechos de otras especies no-humanas y ecosistemas (Haidar y Berros, 2015), y donde las lógicas de mercantilización de los bienes comunes aún prevalecen sobre la capacidad de los Estados de implementar la legislación y dar curso a formas alternativas de relación entre humanos y entornos. La mera declaración no logra implementar efectivamente las propuestas, en tanto no se acompañen de profundos cambios en las sensibilidades, motorizados por la movilización social y articulaciones políticas receptivas a la urgencia de la crisis climática y humanitaria que estamos transitando.

Sostendremos que si bien abren unas vías de indagación más que interesantes al disputar lo establecido por las leyes e instituciones modernas, las visiones ecocéntricas y biocéntricas existentes, materializadas en los DDNN, se encuentran atravesadas por la desigualdad de género. Así, promovemos una mirada más crítica contemplando que se comienza desde una posición desigual y jerárquica -basada en la construcción social del género-, aún subyacente en la formulación discursiva de las leyes y en la amplitud de reconocimientos que efectúa. En este punto sumaremos los aportes del ecofeminismo, que también cuestiona el vínculo depredatorio hacia la Naturaleza desde una sensibilidad y ética afectiva, señalando que existe un correlato entre la dominación de las mujeres y la Naturaleza, constitutiva del modelo civilizatorio heteropatriarcal, colonial y racista. Al proponer una sinergia entre los DDNN y el ecofeminismo, pretendemos ampliar el discurso legal con una práctica teórica y una sensibilidad que lo trascienda.

\section{Los Derechos de la Naturaleza en el marco jurídico moderno}

Los DDNN consolidan una alternativa para el reconocimiento de los seres vivos y entornos como sujetos con derechos. En América Latina consisten, en cierta forma, en un esfuerzo de «traducción» de enfoques de la Pachamama, o la Madre Tierra, presentes en las cosmovisiones de pueblos originarios andinos, en las ideas del sumak kawsay, suma qamaña o el buen 
vivir (Haidar y Berros, 2015; Roa Avendaño, 2009). Especialmente en algunos países, como la República del Ecuador y el Estado Plurinacional de Bolivia, se han expresado visiones pachamámicas en textos constitucionales y leyes. Resulta interesante analizar los procesos por los cuales estas visiones ingresan en la institucionalidad «moderna», y los desafíos para la "traducción» al lenguaje jurídico occidental. Estos esfuerzos no están exentos de tensiones, en gran medida porque resultan una construcción permanente e inacabada de debates, experiencias y movilizaciones acumuladas a lo largo de varios años (Gudynas, 2015).

Lo cierto es que estas conceptualizaciones permiten imaginar nuevos horizontes de sentido sobre los modelos de desarrollo. En la práctica, han logrado cuestionar narrativas modernas y tecnocráticas de la "gobernanza» de la cuestión ambiental, anudadas en el Desarrollo Sostenible (DS) ${ }^{1}$. Al mismo tiempo, los acuerdos internacionales progresivamente han incluido propuestas alternativas basadas en otras experiencias y saberes, aunque como muestran Haidar y Berros (2015) en su estudio del discurso de la «armonía con la naturaleza» promovido por la $\mathrm{ONU}^{2}$, estas fueron subsumidas lentamente en el régimen del ambientalismo neoliberal, esquivando los debates centrales sobre la deuda ecológica entre Norte y Sur globales.

En este punto vale destacar que si bien aquellas experiencias latinoamericanas para reconocer a la Naturaleza como sujeto de derecho representan un avance, demuestran sus límites en el resguardo de grupos humanos y entidades no humanas (tanto ecosistemas como biodiversidad en general) cuando se reproduce el modelo colonial-extractivista. No desconocemos, por supuesto, el gran problema de la dependencia económica, la posición de estos países en la geopolítica internacional y su rol en el sistema-mundo, como proveedores de materias primas y «recursos naturales» ${ }^{3}$.

1 Desde la década de 1970, "la cuestión ambiental" se ha tornado creciente eje de políticas públicas y debates científicos. La salida hegemónica, que desplazó a otras alternativas más críticas o radicales de plantear las desigualdades sociales y ambientales a nivel global, fue el desarrollo sostenible, consolidado en el Informe Brundtland de 1987.

2 Presente en el texto de la Carta de la Naturaleza de 1982. Hacemos notar la tendencia totalizante del discurso del DS, capaz de reducir la amplitud y potencia crítica de los DDNN en un entramado tecnocrático (como un ítem de la dimensión "social" del DS, basado en su perspectiva "multicultural", etc.). Logrando, así, que las concepciones de la Madre Tierra -plurales, biodiversas- mantengan su lugar subordinado.

3 Esto implica no dejar de lado el gran entramado global-financiero en el que adquieren sentido estas realidades de las operaciones extractivas del capital (Gago y Mezzadro, 2015). 


\section{Los límites del modelo}

Se evidencia como un impedimento para los Estados anteponer justicia ambiental por sobre los intereses mercantiles, que condensan un entramado del que participan élites económicas y políticas en un modelo de «maldesarrollo» (Svampa y Viale, 2014). Pueden citarse dos ejemplos paradigmáticos en los países que más hicieron por reconocer a la Naturaleza como sujeto digno de protección: la disputa por el Yasuní en Ecuador y con el TIPNIS en Bolivia ${ }^{4}$ reflejan las tensiones de los gobiernos progresistas cuando persisten en patrones extractivistas, reprimiendo a las comunidades levantadas en defensa de sus territorios contra la expansión de grandes emprendimientos que afectarían sus ecosistemas y medios de vida.

Nuevamente, el reconocimiento formal no alcanza, desde ya, para «torcer» rápidamente los rumbos de las políticas productivas de estos gobiernos. Tampoco es este el lugar para un estudio empírico y exhaustivo que defina esquemas de transición ecológica en forma de DDNN. Sólo ensayaremos algunas reflexiones partiendo de los contextos, y lo haremos asumiendo los márgenes del saber/conocimiento legítimo moderno en que nos movemos. Es importante mencionar que el lenguaje jurídico que efectúa la traducción está asociado a fuertes valores de la modernidad occidental eurocentrista, marcada por dualismos y la matriz simbólica patriarcal. Léase, el lugar del Derecho todavía está marcado por esa legitimidad de la Ciencia, la objetividad, neutralidad, la moral y la racionalidad instrumental. Este es el escenario en que deben jugar los DDNN, e implica tomar esa realidad y operar desde ella, sin dejar atrás valiosos aportes disciplinares de las ciencias y las leyes. Una vez señalado esto, debemos señalar que diversas dimensiones de la desigualdad social nos atraviesan como sociedades modernas y configuran una relación principalmente utilitarista con la naturaleza. Una de ellas es la desigualdad de género ${ }^{5}$.

En 2013, el presidente de Ecuador, Rafael Correa, por decreto pone fin a la "Iniciativa Yasuní-ITT", cuyo objetivo consistía en dejar bajo tierra aproximadamente 850 millones de barriles de petróleo, continuando y acrecentando viejos conflictos con las poblaciones originarias ecuatorianas. Ver: https:// opsur.org.ar/2013/12/10/el-yasuni-en-disputa-apuntes-para-una-cronologia-del-conflicto/ En Bolivia, el conflicto en torno al TIPNIS (Territorio Indígena y Parque Nacional Isiboro Sécure), se desató cuando el gobierno de Evo Morales dispuso la construcción de una carretera, que lo atravesaba por la mitad, en 2007. Ver: https://www.cedib.org/wp-content/uploads/2013/05/El-conflicto-del-TIPNIS-y-sus-implicaciones.pdf

5 Género, racialización y clase son las grandes desigualdades aún imperantes en nuestras sociedades. 
En efecto, y como sucede en otros países de la región, en ambos países los cuerpos feminizados continúan relegados en aspectos tan esenciales como la protección ante distintas formas de violencia y el acceso al mercado formal de empleo. En Bolivia, las mujeres ganan un $47 \%$ menos que los varones y el 61\% de ellas sufrió violencia laboral ${ }^{6}$. En Ecuador el panorama es similar, los salarios de las mujeres son entre un 13 y un $26 \%$ menores que los de sus pares hombres y el $60 \%$ ha experimentado alguna forma de violencia ${ }^{7}$. Con esto queremos destacar algunos aspectos de la estrecha vinculación que existe entre la explotación de la Naturaleza y de los cuerpos feminizados. En este punto, creemos que para alcanzar el buen vivir, reconociendo derechos a sujetos no-humanos y desarmando opresiones y desigualdades entre humanos, resulta imprescindible deconstruir la matriz patriarcal.

En definitiva, nos guía una pregunta: ¿es posible romper con la perspectiva antropocéntrica, que por definición también es androcéntrica, desde la institucionalidad de la ley, sin modificar la estructura patriarcal y su sensibilidad asociada? Orientando las respuestas, el ecofeminismo, como teoría y práctica social, aporta una visión crítica y una sensibilidad alternativa para construir relaciones sociales acorde a otras formas de vincularse con la Naturaleza y entre humanes.

\section{La sensibilidad capitalista-patriarcal}

Para Rita Segato, el género «actúa como una estructura de relaciones entre posiciones, marcadas por un diferencial jerárquico y es una instancia paradigmática de todos los otros órdenes de status: racial, de clase, entre naciones y regiones. El patriarcado es un orden de status, o lo que es lo mismo: un sistema de relaciones marcado por la jerarquía» (Segato, 2010, p. 14). El régimen patriarcal-capitalista requiere de un sistema simbólico que produzca y reproduzca status, jerarquías y explotación de lo vivo. Segato llama «pedagogías de la crueldad» a «todos los actos y prácticas que enseñan, habitúan y programan a los sujetos a transmutar lo vivo y su vitalidad en cosas» (Segato, 2018, p. 13). Esta falta de empatía no es un dato biológico, sino el producto de una construcción social fundada, en primera

6 https://oig.cepal.org/es/documentos/encuesta-prevalencia-caracteristicas-la-violencia-mujeres-2016

7 https://lac.unwomen.org/es/donde-estamos/ecuador 
instancia, en la desigualdad de género. Giraldo y Toro (2020), por su parte, acuñan el término «régimen de afectividad» a lo que "constituye el repertorio sensible que establece los patrones de sensibilidades e insensibilidades, y direcciona las relaciones afectivas en una sociedad. Corresponde a la distribución, selección y gobierno de lo sensible que organiza la experiencia de los cuerpos, estableciendo frente a qué cosas se dirige nuestra sensibilidad; instaurando cuáles elementos se permite amar y ante qué otros permanecer anestesiados, y tutelando el reparto de la economía afectiva y los rieles afectivos de una sociedad» (Giraldo y Toro, 2020, p.124). Para estxs autorxs, la sensibilidad bajo el sistema capitalista moderno impide la apertura empática y la generación de emociones positivas hacia los seres humanos y no-humanos con los que convivimos, convirtiéndolos en cuerpos objetivados y recursos disponibles y explotables (p.58), remarcando que las emociones y sensibilidades están condicionadas por los esquemas sensibles de las sociedades. Estos son una instancia colectiva que nos conforma, «de ahí que el sentipensamiento que da lógica a la explotación, objetivación y devastación de las tramas vitales, está en todo caso guiado por afectos colectivos, por un orden sensible dado por las matrices de significación social» (p. 122).

En esta línea, apuntamos que la matriz patriarcal/androcéntrica consiste en un sistema de opresión de género que fortalece las lógicas de dominación y subalternización, materializadas desde las formas del lenguaje hasta la totalidad de los lazos sociales. Como dice Fabbri, «la masculinidad es un proyecto político extractivista ${ }^{8}$ ya que «busca apropiarse de la capacidad de producción y reproducción de las sujetas a las que subordina. Para que dicho proyecto político sea posible, la masculinidad produce varones deseosos de jerarquía, y pone a su disposición las violencias como medios legítimos para garantizar el acceso a la misma» (Fabbri, 2019, p.56).

La afectación a la naturaleza -y en términos de DDNN, a sus sujetos-, se produce con esas características. El ecofeminismo otorga relevancia al hecho de que el sistema patriarcal es la base que sostiene la subordinación de diferentes grupos humanos (mujeres, lesbianas, travestis, trans, intersex y no binaries, personas racializadas, pueblos indígenas y campesinos) y no-humanos. Debemos pensar las potencialidades de los DDNN teniendo en cuenta estos elementos.

Se refiere a la masculinidad patriarcal. Justamente se plantea la necesidad y posibilidad de crear nuevas masculinidades capaces de expresar otras sensibilidades y generar distintas relaciones sociales. 


\section{Ecofeminismo: modernidad, dualismos y androcentrismo en la socionaturaleza}

En la década de 1970 se produce el encuentro entre el feminismo y el ecologismo. El ecofeminismo reflexiona, explora y activa desde la articulación entre estos dos movimientos, proporcionando claves para situar las contradicciones en las que vivimos, y así revertir los imaginarios dominantes. Como praxis de pensamiento y movimiento social, busca generar nuevas salidas -o recuperar alternativas- de las actuales formas de relacionarnos con la Naturaleza.

Para el ecofeminismo, con la Modernidad se desarrolla y hegemoniza un pensamiento dicotómico que crea constantemente pares de conceptos antagónicos y sexualizados (estereotipos culturales acerca de lo femenino y lo masculino), lo que permite legitimar operaciones de subordinación, al reducir nuestra realidad compleja, multivariada y biodiversa en perspectivas binarias y excluyentes, donde uno de los polos se jerarquiza ante el otro. De esta forma se construye un polo que es feminizado, asociado a la naturaleza, la emoción, lo subjetivo y el cuerpo, y un polo masculinizado, relacionado a lo viril, la cultura, la razón, lo objetivo y la mente. En suma, lo humano (y por ende, la sociedad) fue considerado opuesto a lo natural, y conectado arbitrariamente a una idea de varón ${ }^{9}$ que remite a ciertos estándares: occidental, blanco, burgués y heterosexual. Esta imagen del varón como arquetipo universal sustenta el «pacto androcéntrico» del que habla Maffía (2005, p.20), aquel que pone en el centro de poder a «un varón poderoso, adulto (ni niño ni anciano), propietario, blanco, educado, capaz» (p. 20) y define a todo lo demás como periférico, desvalorado, como alteridad (p.12), habilitando el poder que pone a disposición los cuerpos para su aprovechamiento. Estas consideraciones son claves para pensar las estrategias de los DDNN, en tanto traducción jurídica de enfoques pachamámicos, y donde el Derecho continúa -como el resto de las instituciones modernasasociado a un perfil racional, ilustrado, objetivo (patriarcal).

\section{Leyes y sujetos}

Como señalamos previamente, los DDNN habilitan la disputa de sentidos de lo jurídico y acerca esta esfera a otros dominios de saberes, como

9 Recordemos que varón y mujer son meramente posiciones en un sistema de relaciones y no necesariamente se corresponden con características biológicas del cuerpo humano. 
espacio de encuentro de cosmovisiones y lenguajes territoriales y biodiversos (Haidar y Berros, 2015). Para repensar ese polo jerárquico desde los DDNN, se torna necesario situar el debate en el marco del Estado de Derecho, al cual sostenemos que hay que recuperar de sus limitaciones contractualistas ${ }^{10}$ y moralistas, partiendo de una nueva ética del sujeto corpóreo, necesitado, libre (Hinkelammert y Mora, 2009). Los DDNN también pueden pensarse como una dimensión "ecológica» de la democracia, e incluso se convierten en indispensables, una condición para la realización de los restantes derechos (Acosta, 2008).

Es sobre la ley misma que tenemos que trabajar, en tanto espacio de posibilidades. Como explica Segato, «La ley es un campo de lucha. Su legitimidad depende estrictamente de que contemple desde su estrado un paisaje diverso» (2010, p. 123), ya que "la ley también puede impulsar, informar, sensibilizar ese sentimiento ético y transformar la moral que sustenta las costumbres y el esquema jerárquico» (p. 124). La capacidad discursiva de la ley es su poder simbólico para nombrar y así crear realidades sociales, «Desde la perspectiva de los minorizados, el discurso del Derecho, siempre entendido como un eficaz sistema de nombres en permanente expansión, tiene el poder de agitación, el carácter de propaganda, aún apuntando en la dirección de lo que todavía no existe, que no es aún posible adquirir, en la vida social» (p. 125).

Con la sinergia entre DDNN y ecofeminismo llamamos a interpelar y transformar toda ley o institución que oprime al sujeto humano y nohumano. Esto implica, por un lado, recuperar la primacía de los sujetos por sobre las leyes formales, «el ser humano en cuanto sujeto es el criterio de juicio sobre todas las leyes e instituciones» ${ }^{11}$ (Hinkelammert y Mora, 2009, p.413), y considerar a la vez que los DDNN ingresan como otro crite-

10 El núcleo de este Estado de derecho es el principio de contractualidad, bajo una idea de la ciudadanía compuesta por individuos-propietarios que se relacionan "voluntaria" y "libremente". Estamos hablando fundamentalmente del Estado liberal burgués, "aunque no se presente como tal. Se presenta como Estado de la libertad y la igualdad, lo cual puede ser cierto si entendemos igualdad y libertad como libertad contractual e igualdad contractual (...) La otra cara de esta ley formal de la libertad y la igualdad contractuales es la sociedad capitalista, aun cuando no suele mencionarse explícitamente esta relación con el capitalismo (se la menciona más bien con respecto a la 'economía de mercado')." (Hinkelammert y Mora, 2009, p.409).

11 Como señalan Hinkelammert y Mora (2009, p. 413), recuperando a Marx, nos guía una exigencia a interpelar y transformar toda ley, toda institución, en cuanto ellas humillan, sojuzgan, abandonan o desprecian al ser humano. No es más ni menos que luchar por el respeto a las condiciones de posibilidad de la vida, reconociendo y asumiendo activamente la tensión entre cálculos instrumentales, como las instituciones, y los valores fundamentales para la vida humana (Molina Velázquez, 2008). 
rio de regulación de las instituciones, incorporando a los sujetos no-humanos. Y, por otro lado, añadir una crítica fundamental sobre el patriarcado, como configuración de ese sujeto humano necesitado y corporal. El pensamiento y la crítica ecofeminista se hace imprescindible para integrar la perspectiva de género al discurso de los derechos y empezar a desarmar la lógica de dominio patriarcal sobre los cuerpos y lo viviente en general.

En definitiva, los DDNN abren, articulados con lo que el ecofeminismo devela, nuevos caminos para re-situar las instituciones (ley, mercado) que se nos imponen, que nos oprimen a nosotres y a nuestros entornos -otras especies, ecosistemas-. Para ello debemos retener humildad frente al conocimiento y las acciones humanas, como fragmentarias, parciales, imposibilitadas de abordar toda la totalidad compleja, lo que exige reconocer múltiples saberes y afectos, sin por eso dejar de lado los avances de las diferentes disciplinas científicas y técnicas modernas (Hinkelammert y Mora, 2009; Gudynas, 2018).

\section{Por una política de sensibilidades}

De todo lo anterior queda claro que no podemos perder de vista lo siguiente: en Estados modernos occidentales, la ley como herramienta para forjar nuevas realidades -por ejemplo, reconocer valores intrínsecos a otras especies vivas y ecosistemas- choca no sólo con las formalidades del propio campo jurídico (con todas sus relaciones de poder, normas internas y dispositivos de legitimidad), sino con cuestiones tales como quiénes son los sujetos reconocidos como poseedores de derechos a ser reconocidos y las formas sensibles que la sociedad crea y recrea constantemente. «El sujeto del derecho y la política, el sujeto de la filosofía y la teología, incluso el sujeto de los derechos humanos, es desde la antigüedad hasta la modernidad varón, blanco y propietario» (Maffia, 2005, p.3). En esa línea, para el pensamiento occidental moderno la naturaleza es más bien vista como objeto de dominio y cuidado paternalista. El desafío entonces pasará por «colocar a la naturaleza como sujeto de derecho, quitándole de su ubicación en tanto objeto explotable u objeto a proteger» (Berros, 2015, p.6) insistiendo con una sensibilidad ética "definida como sensibilidad al 'otro', a lo ajeno, y transformada en pivote del movimiento transformador» (Segato, 2010, p. 122).

Producto de la modernidad occidental, el ecofeminismo cuestiona pilares centrales del pensamiento objetivista y neutral, valorando las sensibilidades -el respeto, la comprensión, el cuidado, la compasión, la gratitud, la 
amistad, la responsabilidad- como un elemento insoslayable para las alternativas políticas y todo proyecto democrático y colectivo. Nos resulta un complemento imprescindible para pensar los DDNN desde nuestra propia experiencia vital como hijes de la Modernidad.

Para eso es importante avanzar en la construcción de políticas públicas dirigidas a impulsar el cuidado, en todos los aspectos, desde una perspectiva de género, así como a transversalizar la agenda de las restantes áreas sectoriales del gobierno. Similar trayecto se anticipa para pensar los DDNN, con propuestas que incorporen la visión ambiental amplia y ecofeminista en todos los ámbitos de la función pública, incluyendo la educación ambiental en los distintos niveles de enseñanza, formales y no formales.

En base a lo comentado, coincidimos con algunas apreciaciones de Giraldo y Toro cuando postulan que la lucha política excede la capacidad de instituciones, o la "política tradicional». Para ellxs, el terreno clave de disputa es el campo de la afectividad, «espacio en el que se crean las relaciones narcisistas mercantilizadas, los desafectos, desempatizaciones y la insensibilidad que viabiliza la fuerza de la crueldad. Si no se trabaja en el orden simbólico y afectivo, el viejo orden surgirá, a manera de síntoma, en cualquier nuevo orden, repitiendo sus ecologías ominosas» (Giraldo y Toro, 2020, p.147). Y al mismo tiempo, para potenciar la capacidad de la ley como campo de posibilidades, es necesario desarmar al sistema patriarcal, «trabajar por una reforma de los afectos y de las sensibilidades, por una ética feminista para toda la sociedad» (Segato, 2010, p.131) por ello, es indispensable contar con movimientos sociales, medios de comunicación y leyes capaces de desarmar el discurso hegemónico del capitalismo, empoderar una sensibilidad empática y centrada en la eco-inter-dependencia y dar curso a la creatividad y capacidad de imaginar otros mundos posibles. 


\section{Referencias bibliográficas}

ACOSTA, Y. (2008) Filosofía latinoamericana y democracia en clave de derechos humanos. Editorial Nordan-Comunidad.

BERROS, M. V. (2015) El reconocimiento de derechos a la Pachamama, a la Madre Tierra, y la revalorización de saberes ancestrales en países latinoamericanos: su potencial para la renovación del campo de investigaciones y estudios socio-jurídicos. III Congreso Latinoamericano y Caribeño de Ciencias Sociales Área temática de Desarrollo, Ambiente y Territorio Epistemologías socioambientales: un campo en debate y construcción.

FABBRI, L. (2019) La co-producción de narrativas feministas como método-proceso para el desprendimiento androcéntrico. Tesis para optar por el título de Doctor en Ciencias Sociales. Facultad de Ciencias Sociales, Universidad de Buenos Aires.

GAGO, V Y MEZZADRA, S. (2015). Para una crítica de las operaciones extractivas del capital: patrón de acumulación y luchas sociales en el tiempo de la financiarización, Nueva sociedad, (255), 38-52.

GIRALDO, O. F. Y TORO, I. (2020) Afectividad ambiental: sensibilidad, empatía, estéticas del habitar. Chetumal, Quintana Roo, México: El Colegio de la Frontera Sur. Universidad Veracruzana

GUDYNAS, E. (2015). Derechos de la naturaleza: ética biocéntrica y políticas ambientales. Buenos Aires: Tinta Limón

GUDYNAS, E. (2018). Múltiples paradojas: ciencia, incertidumbre y riesgo en las políticas y gestión ambiental de los extractivismos, Polisemia, 14(25), 5.
HAIDAR, V. Y BERROS, V. (2015) Entre el sumak kawsay y la vida en armonía con la naturaleza: disputas en la circulación y traducción de perspectivas respecto de la regulación de la cuestión ecológica en el espacio global», Theomai, núm. 32, julio-diciembre, pp. 128-150

HINKELAMMERT, F. Y MORA JIMÉNEZ, H. (2009). Economía, sociedad y vida humana: preludio a una segunda crítica de la economía política. Buenos Aires: Editorial Altamira.

MAFFÍA, D. (2005) El contrato moral, en Garrió, E. y Maffía, D. (comp.) Búsquedas de Sentido para una nueva Política. Buenos Aires: Paidós.

MOLINA VELÁSQUEZ, CARLOS (2008). Ética del bien común y de la responsabilidad solidaria" Realidad: Revista de Ciencias Sociales y Humanidades, (117), 365-393. ROA AVENDAÑO, TATIANA. (2009)¿Derecho a la naturaleza o derechos de la naturaleza?, Ecología Política. 38: 17-22.

SEGATO, R. (2010) Las estructuras elementales de la violencia: ensayos sobre género entre la antropología, el psicoanálisis y los derechos humanos. 2a ed. Buenos Aires, Prometeo libros.

SEGATO, R. (2018) Contra-pedagogías de la crueldad. 1a ed. Ciudad Autónoma de Buenos Aires. Prometeo libros

SVAMPA, M. Y VIALE, E. (2014). Maldesarrollo: La Argentina del extractivismo y el despojo. Buenos Aires: Katz editores. 


\section{Pablo Pereira}

Lic. y Prof. en Sociología por la Universidad Nacional de Buenos Aires (UBA). Magister en Conservación de la Biodiversidad (UBA) y Doctorando en Ciencias Sociales (UBA). Docente de Metodología de la Investigación Social y Sociología ambiental en la UBA y la Universidad Nacional de Moreno (UNM). Editor del portal de ecología política Florestanía.

\section{Laura Borsellino}

Lic. en Ciencias de la Comunicación por la Universidad Nacional de Buenos Aires (UBA). Especialista en Conservación de la Biodiversidad (UBA). Periodista ambiental y editora del portal de ecología política Florestanía.

\section{PARA CITAR ESTE ARTícULO}

Pereira Y Borsellino. L. (2021)

ECOFEMINISMOS Y DERECHOS DE LA NATURALEZA. CRUCES ENTRE LEY, ESTADO Y SENSIBILIDADES, en Papeles del Centro de Investigaciones, Facultad de Ciencias Jurídicas y Sociales, UNL, publicación semestral, año 11, número 22, Santa Fe, República Argentina, pp. 59-70. Doi: https://doi.org/10.14409/p. v11i22.10362 